\title{
Range of Motion of the Ankle According to Pushing Force, Gender and Knee Position
}

\author{
Kang Hee Cho, MD, PhD, Yumi Jeon, MD, Hyunkeun Lee, MD
}

Department of Rehabilitation Medicine, Chungnam National University Hospital, Daejeon, Korea

\begin{abstract}
Objective To investigate the difference of range of motion (ROM) of ankle according to pushing force, gender and knee position.

Methods One hundred and twenty-eight healthy adults (55 men, 73 women) between the ages of 20 and 51, were included in the study. One examiner measured the passive range of motion (PROM) of ankle by Dualer IQ Inclinometers and Commander Muscle Testing. ROM of ankle dorsiflexion (DF) and plantarflexion (PF) according to change of pushing force and knee position were measured at prone position.

Results There was significant correlation between ROM and pushing force, the more pushing force leads the more ROM at ankle DF and ankle PF. Knee flexion of $90^{\circ}$ position showed low PF angle and high ankle DF angle, as compared to the at neutral position of knee joint. ROM of ankle DF for female was greater than for male, with no significant difference. ROM of ankle PF for female was greater than male regardless of the pushing force.

Conclusion To our knowledge, this is the first study to assess the relationship between pushing force and ROM of ankle joint. There was significant correlation between ROM of ankle and pushing force. ROM of ankle PF for female estimated greater than male regardless of the pushing force and the number of measurement. The ROM of the ankle is measured differently according to the knee joint position. Pushing force, gender and knee joint position are required to be considered when measuring the ROM of ankle joint.
\end{abstract}

Keywords Articular range of motion, Physical examination, Sex characteristics, Patient positioning

\section{INTRODUCTION}

The ankle joint complex consists of the ankle (talocrural) joint and the subtalar (talocalcaneal) joint. The gross motion between the foot and the shank is the result of the

Received May 26, 2015; Accepted August 24, 2015

Corresponding author: Yumi Jeon

Department of Rehabilitation Medicine, Chungnam National University Hospital, 282 Munhwa-ro, Jung-gu, Daejeon 35015, Korea

Tel: +82-42-338-2460, Fax: +82-42-338-2461, E-mail: asacara@naver.com

(c) This is an open-access article distributed under the terms of the Creative Commons Attribution Non-Commercial License (http://creativecommons. org/licenses/by-nc/4.0) which permits unrestricted noncommercial use, distribution, and reproduction in any medium, provided the original work is properly cited.

Copyright ( 2016 by Korean Academy of Rehabilitation Medicine motions at the ankle joint complex. Because the ankle joint complex is crucial to human locomotion, accurate knowledge on the kinematics of these joints is essential for proper diagnosis and treatment of injuries and diseases in this region [1].

Numerous investigations have been carried out to analyze the kinematic characteristics of the ankle joint complex $[2,3]$. For clinical applications, joint mobility is often quantified by range of motion (ROM), which is clinically defined as the maximal range of joint angle [4].

Studies have proven that there are variations in the degree of mobility of the ankle related to gender and age, in apparently normal individuals $[3,5,6]$. But, most stud- 
ies have been conducted on a Western population [3,79]. There are very few studies regarding the normal ROM of lower extremity joints in the Asian population $[5,10]$. Cultural habits, such as squat toilet use, sitting cross-legged, squatting and kneeling on the ground and religious exercises involving kneeling can affect normal ROM of the ankle joints.

Reproducible measurements of the ROM are an important prerequisite for the interpretation of study results. Visual inspection, goniometric measurements, inclinometer and high-speed cinematography are examples of methods that have been used to quantify the ROM. For this purpose, the digital inclinometer is considered to be a useful instrument due to its cost-effectiveness and ease of use [11].

ROM of ankle can be measured differently by measurement posture, pushing force and gender [12,13]. When the ankle is manually dorsiflexed or plantarflexed, the amount of force applied could influence the amount of ROM achieved $[14,15]$. However, no previous study has assessed adequate pushing force to measure ROM of ankle joint. Furthermore, the extent to which posture of knee joint influences ROM of ankle joint is not well documented. A standardized method for measurement of joint ROM of Koreans has not been presented to date. Korean standard reference for joint ROM and standard and objective method and protocol for measurement of joint ROM are required. The purpose of our study was to evaluate the effect of gender, pushing force and knee joint position on the normal ROM of ankle joint in healthy young Korean subjects.

\section{MATERIALS AND METHODS}

\section{Subjects}

The study included a total of 128 (55 males and $73 \mathrm{fe}$ males) healthy volunteers. Individuals with a history of illness such as inflammatory arthropathy, prior surgery or trauma involving any joint of either lower extremity were excluded from the study. Mean age and age range of the subjects were presented in Table 1.

Table 1. Demographic data of the subjects

\begin{tabular}{lccc}
\hline & Male & Female & Total \\
\hline No. of subject $(\%)$ & $55(42.97)$ & $73(57.03)$ & $128(100)$ \\
Age $(\mathrm{yr})$ & $25.76 \pm 4.7(20-51)$ & $24.99 \pm 3.93(20-36)$ & $25.7 \pm 4.7(20-51)$ \\
BMI $\left(\mathrm{kg} / \mathrm{m}^{2}\right)$ & $22.13 \pm 3.16$ & $21.86 \pm 3.03$ & $22.08 \pm 3.16$ \\
\hline
\end{tabular}

Values are presented as mean \pm standard deviation.

BMI, body mass index.

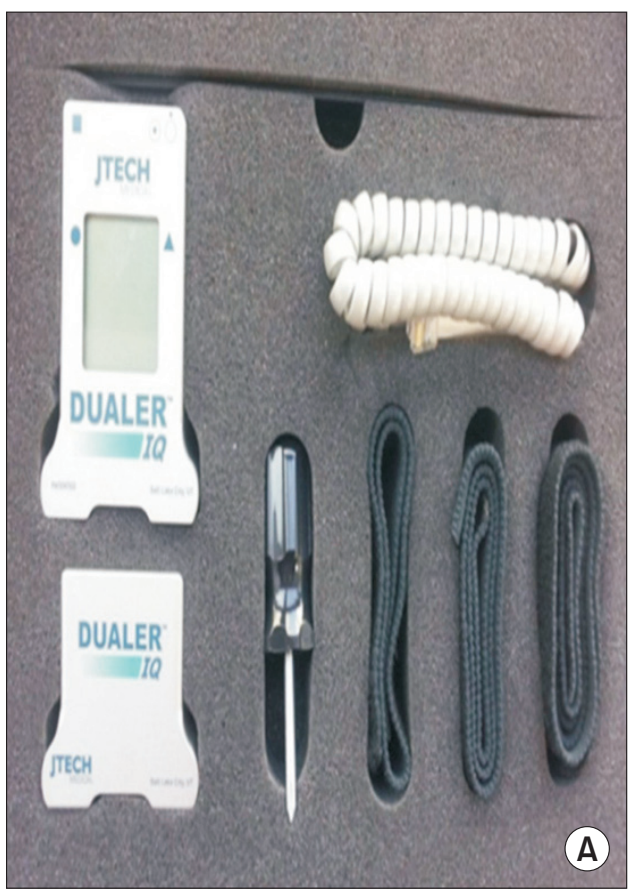

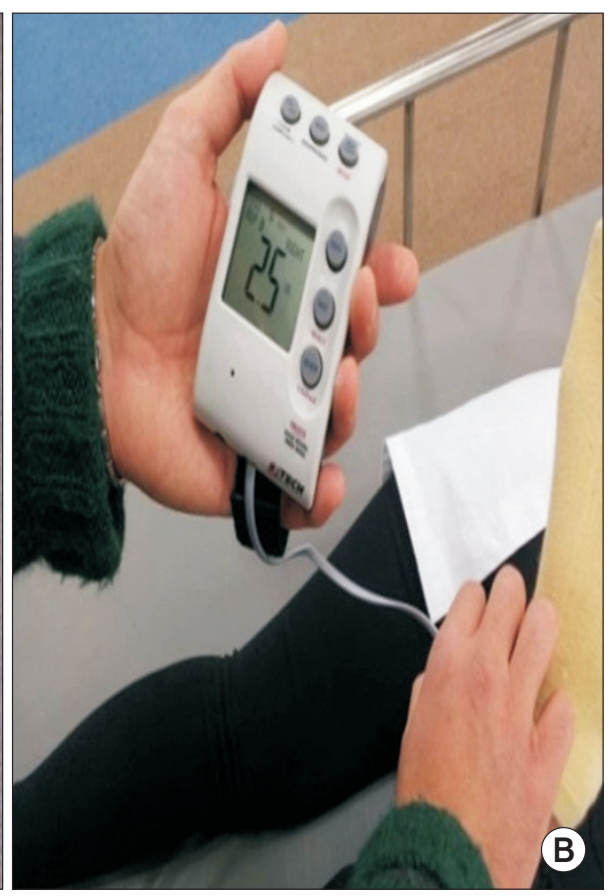

Fig. 1. (A) Dualer IQ Inclinometers (J-Tech, Torrance, CA, USA), (B) Commander Muscle Tester (JTech). 


\section{Methods}

Prior to measuring ROM of ankle, measures of the body mass index (BMI), blood tests including erythro- cyte sedimentation rate (ESR), C-reactive protein (CRP), rheumatoid arthritis (RA) factor and radiologic study of knee \& ankle joint anteroposterior (AP) and lateral, were
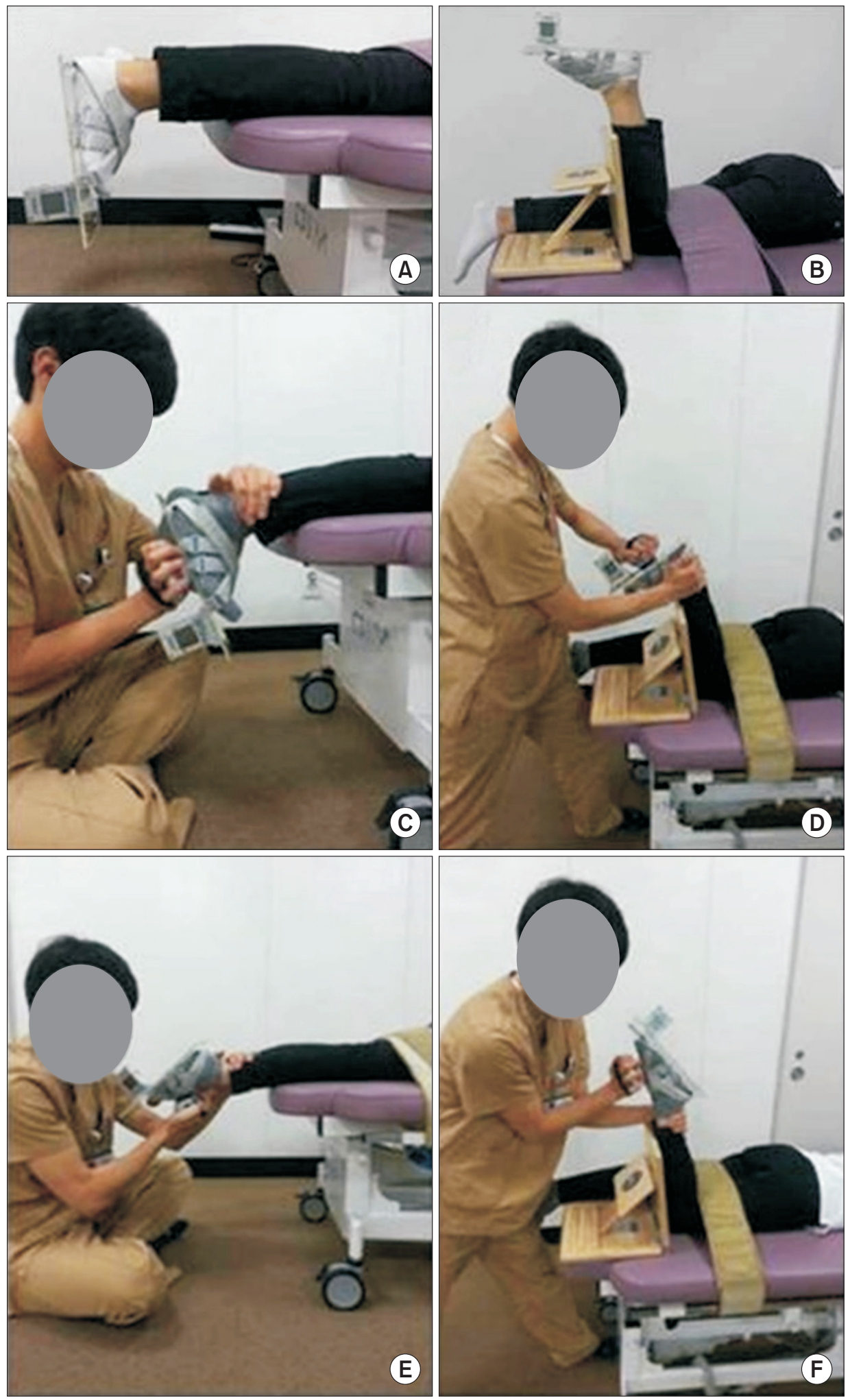

Fig. 2. Starting position before measurement of range of motion of ankle dorsiflexion \& plantarflexion at neutral position of knee joint (A) and ankle dorsiflexion \& plantarflexion at knee flexion of $90^{\circ}(\mathrm{B})$. Position to measurement of range of motion of ankle dorsiflexion (C, D) and ankle plantarflexion (E, F). 
conducted in all subjects, in order to rule out any disease that can affect the ROM of ankle joint such as inflammatory arthropathy, prior surgery or trauma involving any joint of either lower extremity. Subsequently, the examiner completed the ROM measurements. Prior to participation, all procedures were explained to each subject, and informed consent was obtained, as approved by the Institutional Review Board at Chungnam National University Hospital (No. 2014-03-001). The rights of the subjects were protected.

Dualer IQ Inclinometers (J-Tech, Torrance, CA, USA) and Commander Muscle Tester (J-Tech) were used to record the passive ROM of ankle and objective quantify strength (Fig. 1). Prior to actual testing, the inclinometer was calibrated with computer-generated angles to assess accuracy. Next, the examiner established a point of reference by placing the inclinometer on the hand and read the angle while the subtalar joint was maintained in a neutral position. There was perfect agreement between the inclinometer and the reference angles.

One examiner performed ankle ROM measurements using a Dualer IQ Inclinometers and Commander Muscle Tester. Examiner reviewed testing procedures and use of the digital inclinometer prior to actual testing.

ROM of ankle dorsiflexion (DF) and plantarflexion (PF) at neutral position of knee joint and knee flexion of $90^{\circ}$ were measured according to change of pushing force- 0 $\mathrm{kg}$ (full active ROM), $4 \mathrm{lb}$ (1.814 kg), $18 \mathrm{lb}(8.165 \mathrm{~kg}), 31 \mathrm{lb}$
(14.061 kg), $44 \mathrm{lb}(19.958 \mathrm{~kg})$. All measurements were executed with test subjects in prone position with the thigh stabilized to the table with a belt. For the measurement with the knee flexed, the examiner flexed the subject's knee to $90^{\circ}$ and lower leg stabilized to $90^{\circ}$ angle with solid vertical plate (Fig. 2).

The subtalar joint neutral position is considered an important reference position from which motion can be measured. Subtalar joint neutral was determined when the talar head was felt equally between the thumb and index finger. For consistency, examiner determined subtalar joint neutral position as the moment the medial head of the talus was no longer prominent when moving the subject's foot from a pronated to a supinated position. The tester then placed the straight edge of the $\mathrm{Du}$ aler IQ Inclinometers parallel to the 2 dots on the calcaneus while keeping the inclinometer in the frontal plane. During the inclinometer measurement, patients were instructed to hold the position of subtalar joint neutral.

Examiner obtained 5 repeated measures with each method in order to investigate the correlation between passive ranges of motion and these measurements were averaged. Study design consisted of a test-retest crossover with serial measurements for each joint position (Fig. 3).

\section{Statistical analysis}

Statistical analyses were performed using SPSS ver. 18.0 (SPSS Inc., Chicago, IL, USA). Relationships between

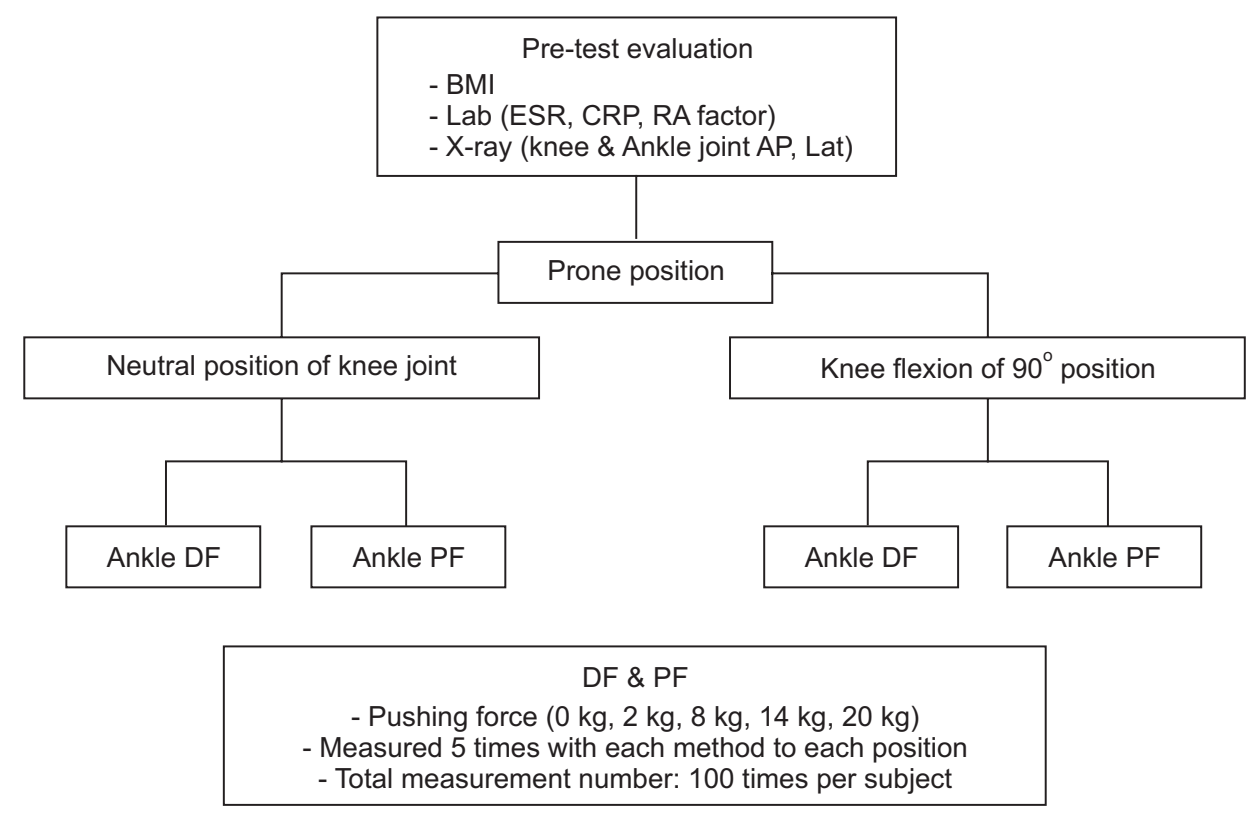

Fig. 3. Overall flow sheet of the measurement process. BMI, body mass index; ESR, erythrocyte sedimentation rate; CRP, C-reactive protein; RA, rheumatoid arthritis; $\mathrm{AP}$, anteroposterior; DF, dorsiflexion; $\mathrm{PF}$, plantarflexion. 
(A)

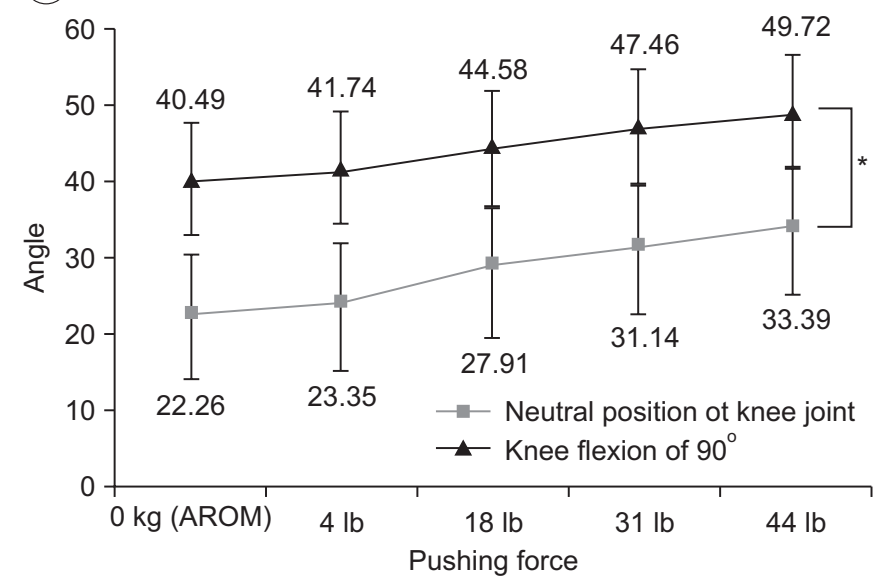

(B)

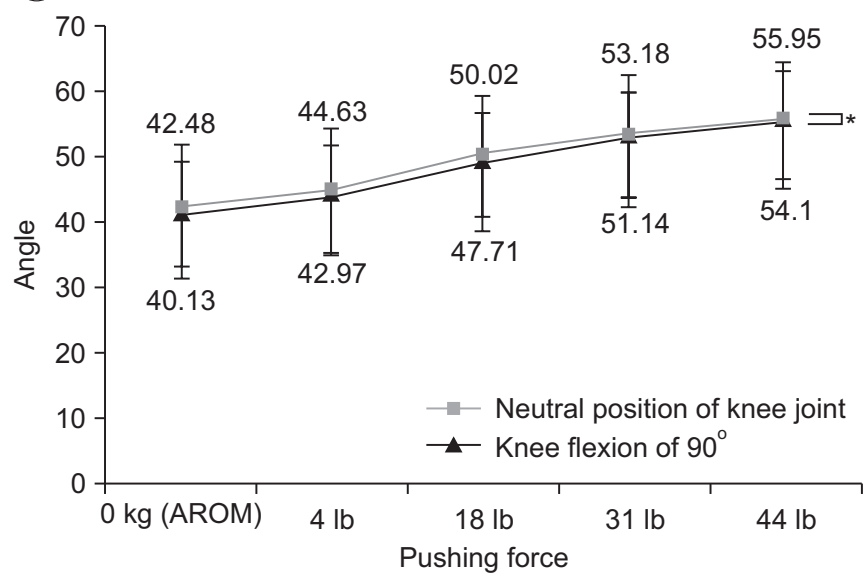

Fig. 4. Measured range of motion (ROM) values of ankle dorsiflexion at neutral position of knee joint and knee flexion of $90^{\circ}$ (A) and plantarflexion at neutral position of knee joint and knee flexion of $90^{\circ}$ (B) associated with pushing force.

pushing force and ROM were calculated using the Pearson correlation coefficient. The independent t-test was used to compare the ROM of male and female subjects and the differences of ankle ROM according to the knee joint position. $p$-values of $<0.05$ were considered significant.

\section{RESULTS}

All 128 subjects enrolled completed the study. Age and BMI showed no significant differences between males and females.

Changes in the measured ROM values associated with pushing force were presented in Fig. 4. There was significant correlation between ROM and pushing force, i.e., the more pushing force leads the more ROM at ankle DF and ankle PF at neutral position at knee joint $(\mathrm{p}<0.05$, $\mathrm{r}=0.993$ and $\mathrm{r}=0.988)$ and at knee flexion of $90^{\circ}(\mathrm{p}<0.05$, $\mathrm{r}=0.998$ and $\mathrm{r}=0.989$ ). There was a significant effect of knee flexion angle on estimated ROM of ankle joint. When compared to the at neutral position of knee joint, knee flexion of $90^{\circ}$ position showed significantly low PF angle and high ankle DF angle $(\mathrm{p}<0.001)$ (Table 2).

ROM of ankle DF for female was greater than male, with no significant difference (Fig. 5). ROM of ankle PF for female was greater than male regardless of the pushing force $(\mathrm{p}<0.05)$ (Fig. 6).

\section{DISCUSSION}

The handbook of the American Academy of Orthope-
Table 2. Relationships between pushing force and ROM of ankle

Correlation coefficient (r)

Neutral position at knee joint
Ankle DF - pushing force
$0.993^{\mathrm{a})}$
Ankle PF - pushing force
$0.988^{\mathrm{a})}$

Knee flexion of $90^{\circ}$

$\begin{array}{ll}\text { Ankle DF - pushing force } & 0.998^{\mathrm{b})} \\ \text { Ankle PF - pushing force } & 0.989^{\mathrm{a}}\end{array}$

ROM, range of motion; DF, dorsiflexion; PF, plantarflexion. ${ }^{a)} \mathrm{p}<0.01,{ }^{\text {b) }} \mathrm{p}<0.001$ by statistical significance using Pearson correlation coefficient.

dic Surgeons (AAOS) is a well-known source for average ranges of joint motions $[4,16]$. Both this handbook and some reports in the literature give no information about the evaluated population (age, gender, race, etc.) or the measurement technic (active or passive) [16,17].

Results of this study identified differences between the mobility of ankle joints in respect to the pushing force, knee joint position and gender in normal Korean individuals. Findings from this study support a simple method using a digital inclinometer to reliably assess ankle ROM in the clinical setting.

The few studies based on Asian populations have shown that ankle DF is significantly greater than those of the Western population $[5,10]$. Kumar et al. [10] reported passive ankle DF of $24^{\circ}$ in the Indian population. In a study with a population of 50 Arab males, Ahlberg et al. [5] reported passive ROMs of $32^{\circ}$ of ankle DF. On the other 
(A)

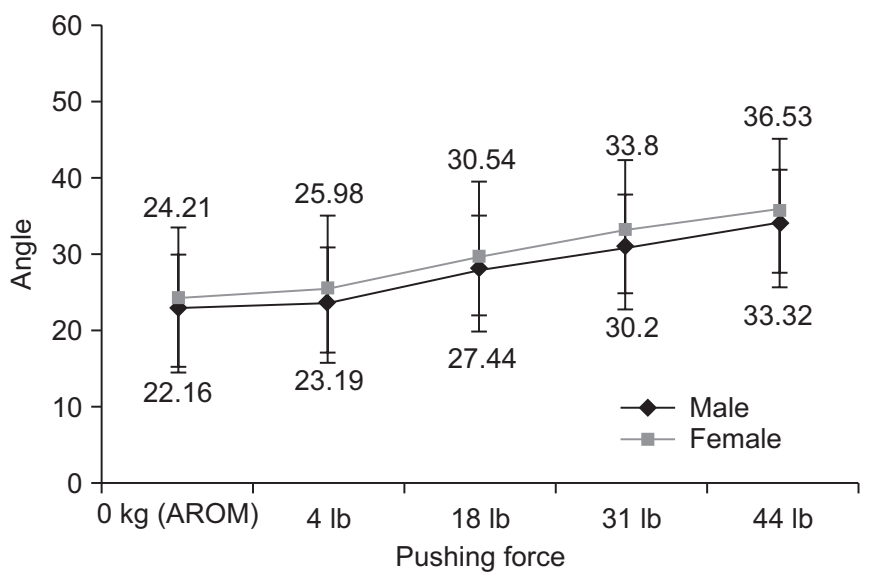

(B)

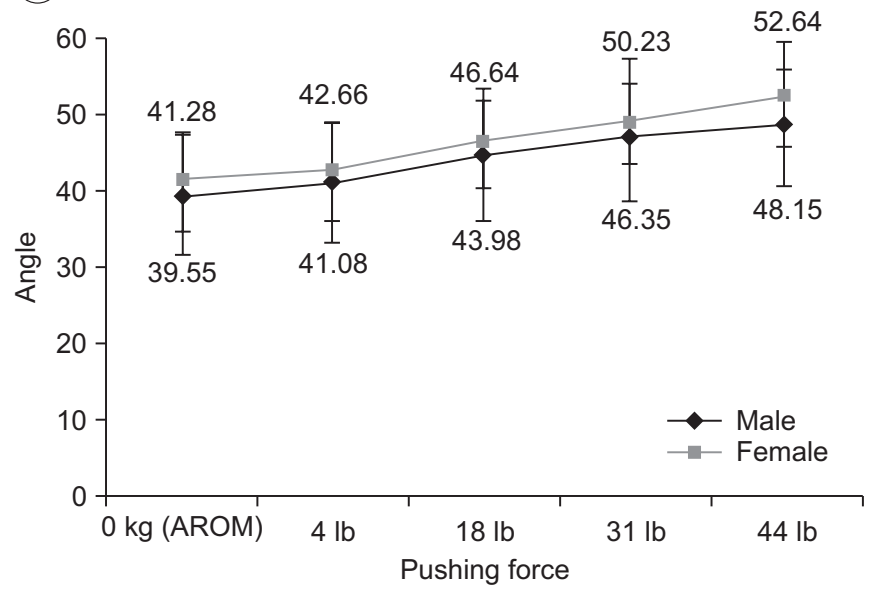

Fig. 5. Differences in the male and female of range of motion (ROM) of ankle dorsiflexion at neutral position of knee joint (A) and at knee flexion of $90^{\circ}(\mathrm{B})$.

(A)

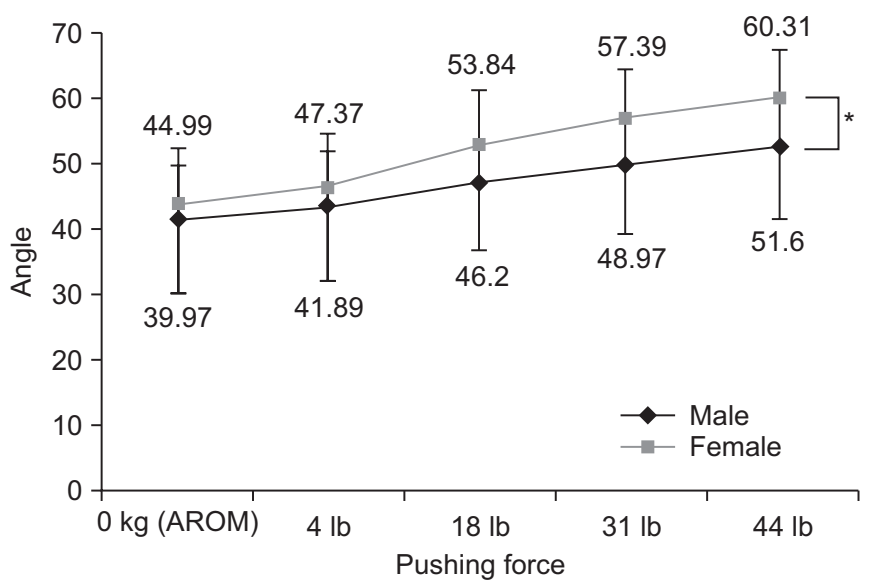

(B)

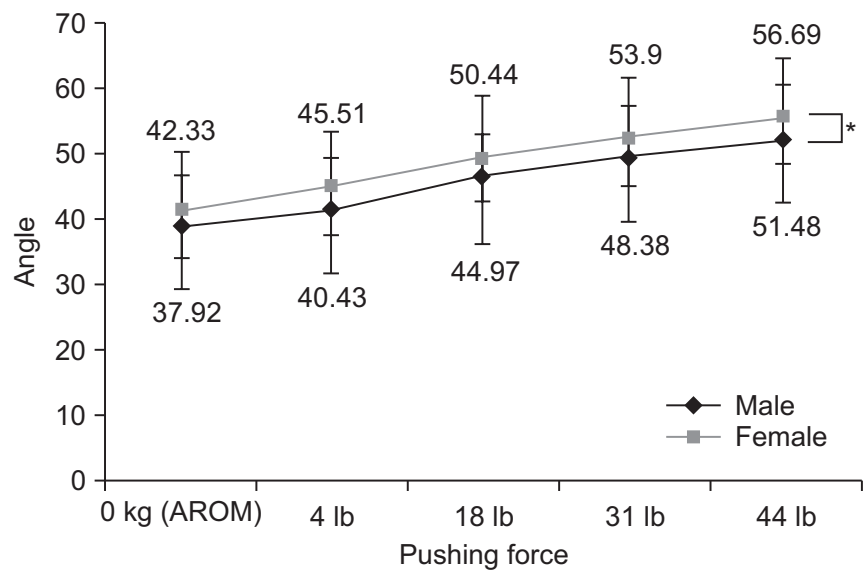

Fig. 6. Differences in the male and female of range of motion (ROM) of ankle plantarflexion at neutral position of knee joint (A) and at knee flexion of $90^{\circ}(\mathrm{B})$.

hand, Roaas and Andersson [3] reported passive ROMs of $15^{\circ}$ of ankle DF in males aged between 30 and 40 years from the city of Gothenburg. The results of this study, in agreement with the previous works $[5,10]$, suggest that Asian populations have shown that ankle DF is significantly greater than those of the Western population.

The mean value for ankle ROM indicate that higher pushing force leads to more ROM at ankle DF and ankle $P F(p<0.05)$. In physics, a force is any interaction that tends to change the motion of an object [17]. Forces act in a particular direction and have sizes dependent upon the strength of push or pull. In other words, a force can cause a change in velocity of an object with mass. Thus the greater the pushing force accelerating the motion of the ankle, leads to increasing of the ROM of ankle. When the pushing force increases, the talocrural joint, as well as with a compensation movement of the subtalar joint and midtarsal joint can lead to larger ROM of the ankle joint. To the best of our knowledge, this study is the first to assess the relationship between pushing force and ROM of ankle joint.

Greater pushing force leads to more ROM at ankle. Therefore, a more standardized manner is required when measuring the joint ROM. It is possible to improve the accuracy of the joint ROM measurement by using a tool like Dualer IQ Inclinometers as used in this study, as 
well as another test with application of the same force. In this study, a wide range of pushing force up to $20 \mathrm{~kg}$, was used to assess the relationship between pushing force and ROM of ankle joint. Since greater pushing force leads to more ROM at ankle, we emphasized the need for new settings to the improvement of the application value and accuracy of the pushing force.

There was a significant effect of knee flexion angle on estimated ROM of ankle joint $(\mathrm{p}<0.001)$. Knee flexion of $90^{\circ}$ position increased the DF angle about $50 \%$, as compared to neutral position of knee joint. Anatomical studies indicate that joint position changes at the knee influence ankle ROM by altering tension within passive tissues that span from the lumbar spine to the foot $[18,19]$. Because the gastrocnemius is a bi-articulate muscle (i.e., crosses both the ankle and the knee), it may be possible to reduce Achilles tendon forces during passive DF simply by flexing the knee. On the other hand, PF had less change associated with the knee joint position. This result considered different synergy effect of other muscles to ankle PF. Further research is required to determine which muscles act as synergist and antagonist of the ankle PF.

In the present study, ROM of ankle PF were higher in female subjects. ROM of ankle DF for female were greater than for male, with no significant difference. Gender differences in the ROM of ankle have been shown for young healthy populations $[20,21]$. A previous study demonstrated that in normal Taiwan individuals, females showed greater joint ROM than males in lower-extremity joints. The greater mobility for female is based on the greater capacity of PF, as compared to male [21]. Our study result of the differences in the male and female corroborated previous descriptions.

A standardized method for measurement of joint ROM of Koreans have not been presented to date. Korean standard reference for joint ROM and standard and objective method and protocol for measurement of joint ROM are required.

A primary goal of healthcare is to understand the boundaries of health and normality and identify when abnormalities are harmful. Diagnosis of disease or impairment is often made by comparing results from clinical measures with healthy reference values. Currently, there is need for comprehensive lower limb reference data representing the healthy population [22]. The 128 normal subjects are currently recruiting to provide reference values for a set of widely-used clinical and biomechanical measures of the ankle ROM.

This study had some limitations. First, this study was performed with participants in young, healthy age group. It is possible that more substantial age-related effects would have become apparent if more participants were tested or if the older cohort was more aged or more frail. Second, in this study, ankle ROM was measured using a digital inclinometer. However, measurement method utilizing the goniometer is more standardized and widely available currently. Comparative ROM measures between these 2 methods would be more meaningful for research purposes and are required in further studies. Third, the patient has to maintain a neutral position of subtalar joint themselves against the larger pushing force, which is very difficult. Therefore, in this study, it may be a significant limitation that we did not use the external device for maintaining the neutral position of subtalar joint during ROM measurement. Fourth, this study is inadequate to determine the most appropriate pushing force to measure the ROM of ankle. Thus, further research is needed that can accurately present the criteria for pushing force to measurement of ROM.

However, this study will contribute substantially to our understanding of the range of normal ankle function in healthy individuals. The reference dataset will be a useful tool for disease diagnosis and management, health surveillance and future outcome measure development for clinical trials of rehabilitative, surgical and pharmacological interventions.

In conclusion, to the best of our knowledge, this is the first study to assess the relationship between pushing force and ROM of ankle joint. There was significant correlation between ROM of ankle and pushing force, i.e., greater pushing force leads to more ROM at ankle DF and ankle PF. ROM of ankle PF for females was greater than for males regardless of the pushing force. According to the knee joint position, the ROM of the ankle is measured differently. Hence, the pushing force, gender and knee joint position should be considered when measuring the ROM of ankle joint.

\section{CONFLICT OF INTEREST}

No potential conflict of interest relevant to this article 
was reported.

\section{ACKNOWLEDGMENTS}

This research was supported (in part) by Ministry of Trade, Industry and Energy through Standard Reference Data Program. We wish to thank Kyun Shik Chae and Chang Geun Kim, the National Center for Standard Reference Data, the Korea Research Institute of Standards and Science, for their general support.

\section{REFERENCES}

1. Wong Y, Kim W, Ying N. Passive motion characteristics of the talocrural and the subtalar joint by dual Euler angles. J Biomech 2005;38:2480-5.

2. Leardini A, O'Connor JJ, Catani F, Giannini S. Kinematics of the human ankle complex in passive flexion; a single degree of freedom system. J Biomech 1999;32:111-8.

3. Roaas A, Andersson GB. Normal range of motion of the hip, knee and ankle joints in male subjects, 30-40 years of age. Acta Orthop Scand 1982;53:205-8.

4. American Academy of Orthopaedic Surgeons. Joint motion: methods of measuring and recording. Edinburgh: Churchill Livingstone; 1966.

5. Ahlberg A, Moussa M, Al-Nahdi M. On geographical variations in the normal range of joint motion. Clin Orthop Relat Res 1988;234:229-31.

6. James B, Parker AW. Active and passive mobility of lower limb joints in elderly men and women. Am J Phys Med Rehabil 1989;68:162-7.

7. Macedo LG, Magee DJ. Differences in range of motion between dominant and nondominant sides of upper and lower extremities. J Manipulative Physiol Ther 2008;31:577-82.

8. Youdas JW, Bogard CL, Suman VJ. Reliability of goniometric measurements and visual estimates of ankle joint active range of motion obtained in a clinical setting. Arch Phys Med Rehabil 1993;74:1113-8.

9. Grimston SK, Nigg BM, Hanley DA, Engsberg JR. Differences in ankle joint complex range of motion as a function of age. Foot Ankle 1993;14:215-22.

10. Kumar S, Sharma R, Gulati D, Dhammi IK, Aggarwal AN. Normal range of motion of hip and ankle in Indian population. Acta Orthop Traumatol Turc
2011;45:421-4.

11. Venturini C, Ituassu NT, Teixeira LM, Deus CV. Intrarater and interrater reliability of two methods for measuring the active range of motion for ankle dorsiflexion in healthy subjects. Rev Bras Fisioter 2006;10:407-11.

12. de Winter AF, Heemskerk MA, Terwee CB, Jans MP, Deville W, van Schaardenburg DJ, et al. Inter-observer reproducibility of measurements of range of motion in patients with shoulder pain using a digital inclinometer. BMC Musculoskelet Disord 2004;5:18.

13. Belcjak CE, Cavalheri G, Pereira JM, Caffaro RA. The differences in the ankle range of motion in distinct ethnical groups measured by goniometry. Phlebologie 2009;38:59-63.

14. Keating JL, Parks C, Mackenzie M. Measurements of ankle dorsiflexion in stroke subjects obtained using standardised dorsiflexion force. Aust J Physiother 2000;46:203-13.

15. Moseley A, Adams R. Measurement of passive ankle dorsiflexion: procedure and reliability. Aust J Physiother 1991;37:175-81.

16. Hallaceli H, Uruc V, Uysal HH, Ozden R, Hallaceli C, Soyuer F, et al. Normal hip, knee and ankle range of motion in the Turkish population. Acta Orthop Traumatol Turc 2014;48:37-42.

17. Kendall FP, McCreary EK, Provance PG, Rodger MM, Romani WA. Muscle: testing and function with posture and pain. 5th ed. Philadelphia: Saunders; 2005. p. 359-464.

18. Mitchell B, Bressel E, McNair PJ, Bressel ME. Effect of pelvic, hip, and knee position on ankle joint range of motion. Phys Ther Sport 2008;9:202-8.

19. Gerlach UJ, Lierse W. Functional construction of the superficial and deep fascia system of the lower limb in man. Acta Anat (Basel) 1990;139:11-25.

20. Cho SH, Park JM, Kwon OY. Gender differences in three dimensional gait analysis data from 98 healthy Korean adults. Clin Biomech (Bristol, Avon) 2004;19:145-52.

21. Chung MJ, Wang MJ. The effect of age and gender on joint range of motion of worker population in Taiwan. Int J Ind Ergon 2009;39:596-600.

22. Baldwin JN, McKay MM, Hiller CE, Nightingale JE, Moloney N, Vanicek N, et al. 1000 Norms Project: understanding foot and ankle health, disease and normality. J Foot Ankle Res 2014;7(Suppl 1):A6. 